\title{
Assessing the Role and Use of Blockchain Technology in the Hospitality and Leisure Industry
}

\author{
Waleed Rashideh
}

\begin{abstract}
Blockchain is an innovative technology, where in the hospitality and leisure industry, travelers can obtain their tourism products and services through an intermediation between travelers and service providers that causes many different problems (e.g. inefficient payment system, extra cost, etc.). The operative effect of blockchain on the hospitality and leisure industry is based on removing the intermediary from the supply chain. Blockchain capabilities are determined according to a number of massive industries, including financial sectors. Such organisations generate various methods in order to simplify trading for smaller and medium sizes of industries. The networks of the tourism value are based on power dependencies' relationships in such a way many expert staff members have acquired additional values that are taken from their partnerships. The structure of a market can be improved based on the value, which relates to different online travel agencies via converting power from suppliers to consumers. The assessment demonstrates that the blockchain technology represents an effective technology that removes mediators, which are originally sourced from the supply chain. This technology does not allow any mediators to gain entry along towards the tourism industry, and by eliminating the market's power.
\end{abstract}

Keywords : Blockchain technology, business model, trustworthiness, third parties, hospitality business.

\section{INTRODUCTION}

$\mathrm{B}$ ockchain Technology (BT) is expected to add $\$ 1.76$ trillion to the global economy by 2030 [1]. It represents a decentralised database approach that is accompanied by increased performances and a robust security system for protecting different types of data [2]. Moving ahead towards the current disruptive technology represents different disruptive modifications related to an industry through various practices [3]. When this technology is being used within the tourism industry, travellers' physical IDs will no longer be required to be presented during their travelling process, and this puts a positive impact on such a process. Furthermore, using such a technology can assist different industries and organisations in protecting digital records along with the protection of the stored data.

The technology integrates transactions and data, which are

Manuscript received on August 15, 2021.

Revised Manuscript received on September 01, 2021.

Manuscript published on August 30, 2021.

* Correspondence Author

Waleed Rashideh*, Department of Information System, College of Computer and Information Sciences, Imam Muhammad Ibn Saud Islamic University, Riyadh, Saudi Arabia. Email: wmrashideh@imamu.edu.sa

(c) The Authors. Published by Blue Eyes Intelligence Engineering and Sciences Publication (BEIESP). This is an open access article under the CC BY-NC-ND license (http://creativecommons.org/licenses/by-nc-nd/4.0/) registered and transformed through a particular a network related to different disseminated ledgers [4]. The aspect of this technology is proposed by Nakamoto [5] based on applying different cryptocurrencies approaches. In fact, the technology functions with no any centralised authority. [6][7]. Applying different cryptographic algorithms implies that each connected node to a network obtains an increased security level during the validation of different transactions [5][7][8]. Many different technologies are being improved and are applied so that the BT technology turns to be more efficient and robust. For instance, the smart contract attempts to simplify the use of high disintermediation levels. Hence, the benefits of the technology can be taken into account within the tourism industry. This review will shed the light on how blockchain technology will transform the hospitality industry.

\section{THE IMPORTANCE OF BLOCKCHAIN TECHNOLOGY FOR TOURISM INDUSTRY}

A few companies have considered the BT technology by proposing different approaches for operating their tasks. The tourism industry is based on its diversification and the complicated business relationships, which involves many business processes, partners, travel services and individuals when a competition gets higher than ever. This requires that such processes should be appropriate to the entire involved parties [9]. Decentralised applications (DApps) have been improved and involved with blockchain in order to satisfy consumers within the tourism industry [10], particularly, based on different smart destinations [11]. Online users can redesign the tourism industry when generating a few companies and ignoring other unrequired ones [12]. These online reviews are made reliable to novice users since they are genuine [13]. There exist many views on the effect of the BT on the hospitality and leisure area where a positive effect on different review systems may assist in obtaining reliable rating approaches towards travelers when either performing or amending their decisions regarding their intended tourism destinations [14]. Lacking appropriate protocol rules implies that personal reputations are seen to be further significant in terms of business interactions. Accordingly, cryptocurrencies simplify a customer-to-customer transaction, which is currently proposed to many secondary and primary markets related to tourism services and products [15][16] . The power of the BT is based on acquiring different positive influences on business agreements [17].

Published By:

Blue Eyes Intelligence Engineering and Sciences Publication

(C) Copyright: All rights reserved.

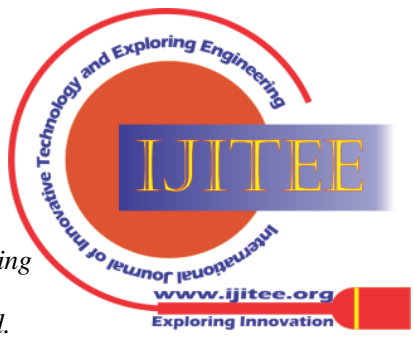


Proposing to introduce Bitcoin as a cryptocurrency has currently altered the way individuals perceive on the BT [18] as there is no reliability on banking systems [19]. Hence, organisations and consumers are motivated to seek appropriate solutions for businesses [18] [19]). The most essential comparison between the blockchain-based intermediaries and the existed business model pertaining to the online travel agencies refers to the fact that cryptocurrencies are successfully combined through their business models [20]. Further, the blockchain adoption relies on the new technology awareness and knowledge levels [20] [21].

\section{BLOCKCHAIN TECHNOLOGY IMPLEMENTATION}

Before Travelers must demonstrate their identifications according to many different steps throughout their journeys. Based on the BT, this process is made easier and further secured by displaying the identification of every traveler once only. The BT can monitor the luggage's movements during travelling when applying a decentralised database among many organisations [22].

Tourism companies have launched the use of the BT in order to reserve and pay for different required bookings and further activities related to their clients' travel [23]. In fact, this action provides the permit for travelers to make their bookings by applying different cryptocurrencies. By the end of 2020, CoinMarketCap recorded over 8,164 cryptocurrencies, including the market cap that reached about $\$ 893$ billion of the bitcoin dominance, which represents $69 \%$ of the market cap (https://coinmarketcap.com/). A few blockchains are applied by a platform within the tourism industry. For instance, LockChain attempts to rent a property and BeeToken / Beenest attempts to rent a Home-sharing platform [9] [22]. Figure 1, depicts the current and future expectations pertaining to the blockchain investment that is distributed by an industry. Tourism industry has a great potential of these investments.

\section{Blockchain investment by industry}

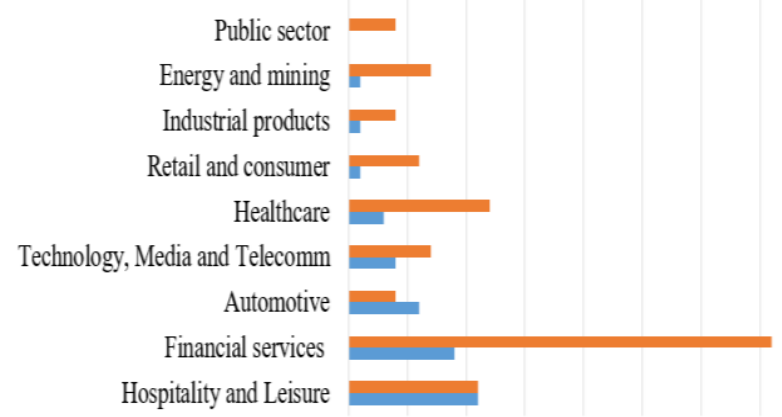

$$
\begin{array}{llllllllll}
0 \% & 5 \% & 10 \% & 15 \% & 20 \% & 25 \% & 30 \% & 35 \% & 40 \%
\end{array}
$$

FIGURE 1. Summary of blockchain investment by industry Source: [24]

o functionality of blockchain technology

Opportunities are acquired to adopt the BT in many organisations [25] where this technology will gain a positive influence on many other organisational concepts [26]. The $\mathrm{BT}$ is used to solve the problem of trust in online reviews [27] as it provides a reliable environment since it is transparent and immutable, and hence, ensuring the integrity of the data [28], preventing manipulation [29]. The most important advantages of applying the BT to several sectors in smart cities where they note that the technology is characterised by trust, freedom, decentralisation, security, transparency and automation [30]. Other recently identified advantages are immutability, low cost and traceability [31]. Before using the blockchain in the development of applications, it is necessary to choose an appropriate type of blockchain, whether a public or private type or a consortium, based on the needs of the applications. A public blockchain is characterised by having no central authority [32]. A consortium blockchain is considered partially decentralised [26]. In this type of blockchain, a number of nodes are assigned to specifically perform the verification and control processes [32]. A private blockchain is characterised by a central network [32] that is operated by one organisation, which includes a number of nodes that can verify and access its data [26].

o The potential business model of blockchain

Organisations must be aware of the changes, which are applied within a business model in order to benefit from the BT. Additionally, applying this technology needs a substantial collaboration among tourism stakeholders [33][34].The entire sectors related to the tourism and hospitality industry are influenced by different innovations in the BT [25]. Blockchain is applied in travel agencies, food services and in accommodation sectors. For instance, accommodation sectors involve financial management, revenue management, and guest history and inventory control [25]. Smart contract will improve and shape the new business model. Smart contracts are agreements that imposed on and applied to unknown parties that implement and perform transactions without any central control [35] [36]. In addition, these types of contracts are suitable for implementation in many areas and are concerned on consistency and aims to provide an analysis of the features of the protocols for achieving consistency within the blockchain technology [37]. Trust has also played an important role in encouraging companies and individuals to be ready to accept blockchain through its entire applications and uses. Privacy and security are the most significant elements of trust [38]. The role of trust might have an impact on the type of changes, which could produce a blockchain cause into the business model. Therefore, blockchain is considered as a business model innovation within the tourism industry.

\section{THE PROBLEM SOLVING BY BLOCKCHAIN TECHNOLOGY}

Many initiatives attempt to connect stakeholders with each other, and solve the issues related to market entry by eliminating the third parties [14]. This yields to save costs for consumers and producers [6]. Peer-to-peer business models are enhanced based on the BT where some factors are improved such as the shared economy (e.g., Uber) [39]. The BT can deactivate the new model of the tourism industry.

Blue Eyes Intelligence Engineering and Sciences Publication

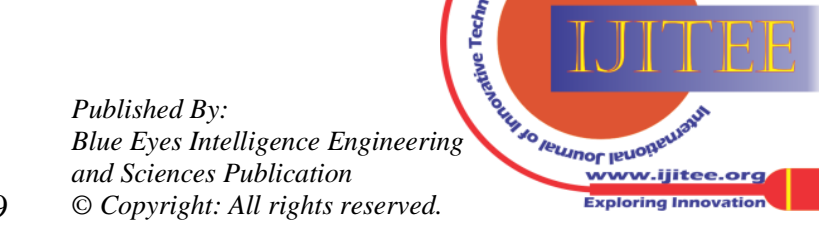


There are some companies as Airbnb, Lyft and Uber, which are intermediaries for ensuring the reliability of their apartment landlords or own drivers. Additionally, intermediaries aim to generate an economic value among themselves [40]. Nonetheless, they are not frequently reliable. Minimising the demand for intermediaries is based on complying with the trends of the BT [41]. Hence, blockchain must not rely on intermediaries of many different fields as in the business sector [42][43]. In this paper, a review study regarding the $\mathrm{BT}$, including its future effects on the tourism industry is highlighted. The objective is to enhance a blockchain-based method for increasing the disintermediation level within the prospective industry and the role of the BT within the tourism industry. It is found to be proven from the findings that the BT can support eliminating any current intermediaries from the tourism supply chain by improving a method, which increases the disintermediation level. There is an urge need for a method, which can minimise the cost and time related to the tourism-related travel. Furthermore, securing the data of travelers requires further reliable method that can operate a tourism business. It is recommended to apply such a technology for incrementing the disintermediation level within the tourism industry based on the reduction of any inefficiencies and by incrementing the data security within the prospective industry.

\section{DISCUSSION AND CONCLUSION}

Blockchain technology conducts an efficient performance within the tourism industry since travelers can take advantage from applying it into their needs. The features of blockchain involve immutability, which can render auditing a rapid, cost-effective and effective process by obtaining integrity and confidence for the data in different organisations. Blockchain provides an increased security level for an individual user as it attempts to remove using an easy-to-hack and weak identities and passwords throughout the internet. On the other hand, transparency implies that each user within the blockchain has full knowledge of the transactions being involved. Additionally, there exist different benefits in privacy, more rapid business procedures, traceability, cost minimisation from decentralisation and automation. Furthermore, such a technology can be a benefit that is based on many concepts within the travel industry (e.g. reliability, security, new business model, performance, money-transfer applications, etc.). This review can assist many practitioners within the travel industry in order to make them able to identify the practicality and benefits of the BT. Depending on many different intermediaries is significant for several individuals and this refers back to many reasons. Consequently, it is recommended to integrate the blockchain into the tourism business models for minimising the number of intermediaries and the current entrants within an organisation.

\section{REFERENCES}

1. G PwC. Time for trust: How blockchain will transform business and the economy. Retrieved from: https://www.pwc.com/gx/en/industries/technology/publications/bloc kchain-report-transform-business-economy.html. (2020).
2. Khalifa. Blockchain: The UPcoming Revolution in the World of Management And Finance. Futureuae. Retrieved from: https://futureuae.com/media/Ehabpdf_d1f747f1-7ba7-4390-bd3f-918 c5dbf6ead.pdf. (2018).

3. S. Kurpjuweit et al, "Blockchain in additive manufacturing and its impact on supply chains," Journal of Business Logistics, 2019.

4. T. Felin and K. Lakhani, "What problems will you solve with blockchain?" MIT Sloan Management Review, vol. 60, (1), pp. 32-38, 2018.

5. Nakamoto, S. (2008). Bitcoin: A peer-to-peer electronic cash system.

6. F. Hawlitschek, B. Notheisen and T. Teubner, "The limits of trust-free systems: A literature review on blockchain technology and trust in the sharing economy," Electronic Commerce Research and Applications, vol. 29, pp. 50-63, 2018.

7. M. C. Lacity, "Addressing key challenges to making enterprise blockchain applications a reality," MIS Quarterly Executive, vol. 17, (3), pp. 201-222, 2018.

8. D. Drescher, "Blockchain Basics: A Non-Technical Introduction in 25 Steps (2017)," Daniel Drescher, .

9. S. Melnychenko, N. Mazaraki and T. Tkachuk, "Leading trends in tourism: Blockchain in franchising," in 3rd International Conference on Social, Economic, and Academic Leadership (ICSEAL 2019), 2019,

10. H. Kennedy-Eden and U. Gretzel, "A taxonomy of mobile applications in tourism," 2012.

11. S. Khan. 3 Ways blockchain can boost brand marketing, Adweek. (2018).

12. M. Bassig, "No title," Hoteliers and Tourism Business Owners Travel, Tourismand Hotel Review Sites You should Monitor, 2012.

13. R. Filieri, "What makes an online consumer review trustworthy?" Ann. Tourism Res., vol. 58, pp. 46-64, 2016.

14. I. Önder and H. Treiblmaier, "Blockchain and tourism: Three research propositions," Ann. Tourism Res., vol. 72, (C), pp. 180-182, 2018.

15. D. Calvaresi et al, "Trust in tourism via blockchain technology: Results from a systematic review," in Information and Communication Technologies in Tourism 2019Anonymous 2019,

16. G. Gu and F. Zhu, "Trust and disintermediation: Evidence from an online freelance marketplace," Management Science, 2020.

17. M. Valeri, "Blockchain technology: Adoption perspectives in tourism," in Entrepreneurship and Organizational ChangeAnonymous 2020.

18. B. Marr, "A short history of Bitcoin and crypto currency everyone should read," Dostupno Na Https://Www.Forbes.Com/Sites/Bernardmarr/2017/12/06/a-Short-Hi story-Ofbitcoin-and-Crypto-Currency-Everyone-should-Read, 2017.

19. B. Yandle, "Lost trust: The real cause of the financial meltdown," The Independent Review, vol. 14, (3), pp. 341-361, 2010.

20. L. Deloitte, "Blockchain Enigma. Paradox. Opportunity," The Creative Studio at Deloitte, London J, vol. 7969, 2016.

21. B. Marr, "The 5 big problems with blockchain everyone should be aware of," Forbes, vol. 19, pp. 2018, 2018.

22. M. Kizildag et al, "Blockchain: A paradigm shift in business practices," International Journal of Contemporary Hospitality Management, 2019.

23. E. Sixtin, " TUI Tourism Group Will Adopt Ethereum Blockchain Technology," TUI Tourism Group Will Adopt Ethereum Blockchain Technology, 2017.

24. PwC,. Global Digital IQ Survey. Retrieved from: https://www.pwc.com/us/en/library/digital-iq.html . (2017)

25. P. Willie, "Can all sectors of the hospitality and tourism industry be influenced by the innovation of blockchain technology?" Worldwide Hospitality and Tourism Themes, 2019.

26. M. Niranjanamurthy, B. N. Nithya and S. Jagannatha, "Analysis of Blockchain technology: pros, cons and SWOT," Cluster Computing, vol. 22, (6), pp. 14743-14757, 2019.

27. N. Emmadi, L. P. Maddali and S. Sarkar, "Marschain: Framework for a fair manuscript review system based on permissioned blockchain," in European Conference on Parallel Processing, 2018,

28. S. Seebacher and R. Schüritz, "Blockchain technology as an enabler of service systems: A structured literature review," in International Conference on Exploring Services Science, 2017,

29. R. Ramachandiran, "Using Blockchain Technology To Improve Trust In eCommerce,"

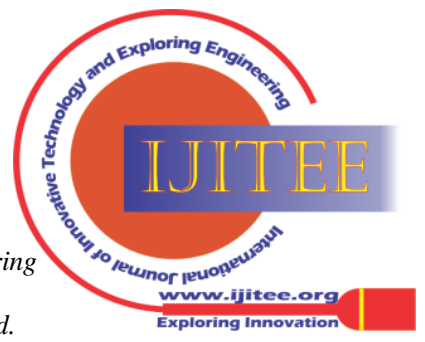


30. J. Xie et al, "A survey of blockchain technology applied to smart cities: Research issues and challenges," IEEE Communications Surveys \& Tutorials, vol. 21, (3), pp. 2794-2830, 2019.

31. [31] K. Nam et al, "Blockchain technology for smart city and smart tourism: latest trends and challenges," Asia Pacific Journal of Tourism Research, pp. 1-15, 2019.

32. L. S. Sankar, M. Sindhu and M. Sethumadhavan, "Survey of consensus protocols on blockchain applications," in 2017 4th International Conference on Advanced Computing and Communication Systems (ICACCS), 2017,

33. R. Böhme et al, "Bitcoin: Economics, technology, and governance," Journal of Economic Perspectives, vol. 29, (2), pp. 213-238, 2015.

34. A. O. Kwok and S. G. Koh, "Is blockchain technology a watershed for tourism development?" Current Issues in Tourism, vol. 22, (20), pp. 2447-2452, 2019.

35. R. Cheng et al, "Ekiden: A platform for confidentiality-preserving, trustworthy, and performant smart contracts," in 2019 IEEE European Symposium on Security and Privacy (EuroS\&P), 2019, .

36. S. Wang et al, "Blockchain-enabled smart contracts: architecture, applications, and future trends," IEEE Transactions on Systems, Man, and Cybernetics: Systems, vol. 49, (11), pp. 2266-2277, 2019.

37. L. Kiffer, R. Rajaraman and A. Shelat, "A better method to analyze blockchain consistency," in Proceedings of the 2018 ACM SIGSAC Conference on Computer and Communications Security, 2018,

38. D. D. Shin, "Blockchain: The emerging technology of digital trust," Telematics Inf., vol. 45, pp. 101278, 2019.

39. S. Huckle et al, "Internet of things, blockchain and shared economy applications," Procedia Computer Science, vol. 98, pp. 461-466, 2016.

40. T. Kohler, A. Stribl and D. Stieger, "Innovation for volunteer travel: Using crowdsourcing to create change," in Open TourismAnonymous 2016, .

41. D. Tapscott and A. Tapscott, Blockchain Revolution: How the Technology Behind Bitcoin is Changing Money, Business, and the World. 2016.

42. W. Rashideh, "Blockchain technology framework: Current and future perspectives for the tourism industry," Tour. Manag., vol. 80, p. 104125, Oct. 2020.

43. D. Banerji, R. Waleed, B. Arora, and A. Pratihari, "Application potential of Blockchain technologies in the travel and tourism industry," in Blockchain Applications in IoT Ecosystem, 2021, pp. 289-299.

\section{AUTHORS PROFILE}

Waleed Rashideh, is Assistant Professor of information system with extensive background of developing and implementing special programs, working at the College of computer and information Sciences, Imam Mohammad Ibn Saud Islamic University, KSA. He has received his doctorate degree from the University of Science Malaysia/ Malaysia. He is author of severa publications in international refereed journals. His research interests include Blockchain Technology, Project Management, and Business Intelligence.

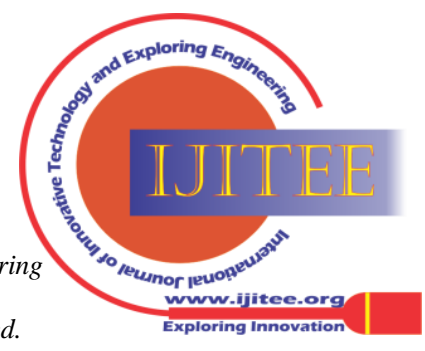

